\title{
Comportement dynamique des lignes hydrauliques de transmission de puissance
}

\author{
Dynamic behaviour of hydraulic power transmission lines
}

\author{
Jacques Lallement \\ Ingénieur Service Hydraulique CETIM
}

\section{Introduction}

L'amplitude importante des pulsations ou des phénomènes instationnaires rencontrés dans les canalisations peut nuire au bon fonctionnement d'un circuit hydraulique en créant des instabilités. D'une manière plus générale, il peut y avoir des surcharges et une fatigue importante entraînant la rupture du matériel.

Ces pulsations et instabilités sont des sources de vibration excitant les structures mécaniques et sont des générateurs de bruit.

Pour les raisons énoncées ci-dessus, il s'avère nécessaire de prévoir la génération, la transmission et l'atténuation des pulsations de pression et de débit dans les canalisations pour fixer les règles qui permettront de réduire l'importance des phénomènes instationnaires parasites dans les transmissions hydrauliques. C'est le but de l'étude présentée.

De nombreux travaux ont déjà été réalisés sur des circuits hydrauliques particuliers avec excitations bien définies (de type échelon ou périodique). L'apport de cette étude a été de mettre au point une méthode de calcul des régimes transitoires dans les canalisations fluides valables pour tout appareil placé en extrémité et pour tout signal d'excitation en tenant compte de l'amortissement propre du fluide.

L'intérêt industriel pour la profession des transmissions hydrauliques est la prévision de tout phénomène vibratoire d'origine fluide nuisant au bon fonctionnement et à la durée de vie du matériel. Les résultats de cette étude peuvent être appliqués dans d'autres domaines de la technique tels que l'hydraulique industrielle, l'étude de l'écoulement du sang dans les artères, l'étude des circuits de graissage, pourvu que l'on puisse considérer que le fluide est newtonien, l'écoulement laminaire et la déformation des canalisations parfaitement élastique.
A partir des équations de base de la mécanique des fluides et d'hypothèses simplificatrices, le CETIM a développé une théorie sur les phénomènes transitoires ; cette théorie a abouti à une méthode de calcul qui peut être utilisée, quelles que soient les variations avec le temps des pressions ou débits aux extrémités de la ligne, indépendamment de la nature des organes placés à l'entrée et à la sortie.

\section{Notations - Symboles}

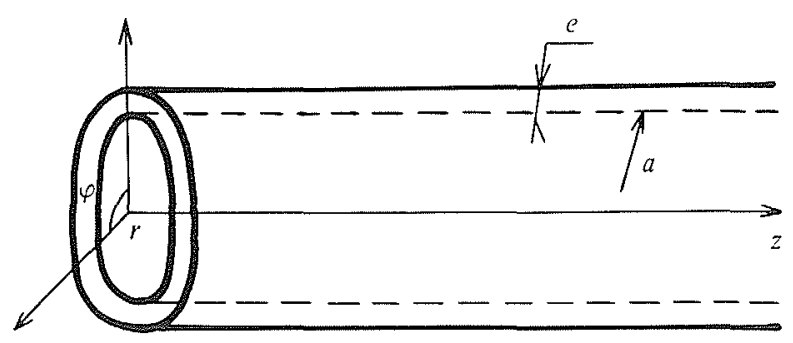

en coordonnées polaires:

$$
\left\{\begin{array}{l}
r \\
\varphi \\
z
\end{array}\right\} \quad \vec{v}=\left\{\begin{array}{l}
u \\
v \\
w
\end{array}\right\}
$$

$\begin{array}{ll}t & \text { temps } \\ w(z, r, t) & \text { vitesse axiale } \\ p(z, t) & \text { pression statique dans une section droite } \\ q(z, t) & \text { débit masse dans une section } \\ \rho & \text { masse volumique } \\ K & \text { module de compressibilité isotherme } \\ K u e & \text { module de compressibilité équivalent } \\ \theta & \text { température absolue } \\ \alpha & \text { coefficient de dilatation thermique } \\ C_{p} & \text { chaleur spécifique } \\ L & \text { longueur de la canalisation }\end{array}$


$\gamma \quad$ viscosité cinématique

a rayon intérieur de la canalisation

$\alpha \quad$ section intérieure droite de la canalisation

$e \quad$ épaisseur de la canalisation

$\tau$ paramètre d'amortissement

$T \quad$ temps de propagation

$c=\sqrt{K e / \rho}$ vitesse du son équivalente

$E \quad$ module élastique

$\bar{t} \quad$ temps réduit

$s \quad$ variable de Laplace par rapport au temps $t$

$\bar{s} \quad$ variable de Laplace par rapport au temps $\bar{t}$

A.B.C. fonctions caractéristiques

Toutes les unités utilisées sont celles du système SI

\section{Etude théorique}

\section{Eúude du modèle physique}

L'étude de la dynamique d'un fluide visqueux sous pression, enfermé dans une enveloppe déformable est régie par cinq équations moyennant les hypothèses simplificatrices suivantes (cf réf. 1)

- le fluide est newtonien et l'écoulement laminaire

- le fluide est animé de mouvements axiaux de vitesse faible par rapport à la célérité du son

- les constantes physiques du fluide, viscosité, compressibilité sont supposées ne pas être affectées par les variations de la pression et de la température.

- la déformation de la tuyauterie est supposée parfaitement élastique.

Les cinq équations sont :

- l'équation d'état du fluide

$$
d \rho / \rho=-\alpha d \theta+d p / K
$$

- l'équation de continuité

$$
\frac{\partial}{\partial t}(\rho \mathcal{Q})=-\frac{\partial q}{\partial z}
$$

- 1'équation de déformation de la tuyauterie

$$
d \mathcal{Q} / \mathcal{Q}=2 a / E / e \cdot d \rho
$$

- l'équation du mouvement du fluide

$$
\frac{\partial w}{\partial t}=-\frac{1}{\rho} \frac{\partial \rho}{\partial z}+\nu\left(\frac{\partial^{2} w}{\partial r^{2}}+\frac{1}{r} \frac{\partial w}{\partial r}\right)
$$

- l'équation de l'énergie

$$
\frac{\partial \theta}{\partial t}=\frac{\alpha \theta}{\rho C_{p}} \frac{\partial p}{\partial t}
$$

Ces cinq équations se réduisent pour donner les deux équations suivantes:

$$
\left\{\begin{aligned}
& \frac{\rho}{K e} \frac{\partial p}{\partial t}+\frac{\partial q}{\partial z}=0 \frac{1}{K e}=\frac{1}{K}-\frac{\alpha^{2} \theta}{\rho C_{p}}+\frac{2 a}{E e} \\
& \frac{\partial^{2} w}{\partial r^{2}}+\frac{1}{r} \frac{\partial w}{\partial r}-\frac{1}{\gamma} \frac{\partial w}{\partial t}-\frac{1}{\rho \nu} \frac{\partial p}{\partial z}=0
\end{aligned}\right.
$$

En utilisant la transformation de Laplace des équations (6) et (7), en intégrant (7) par rapport aux variables $r$ et $z$, on montre que l'on obtient (cf réf. 1), l'égalité matricielle suivante qui lie les variations de pressions et de vitesse aux extrémités.

$$
\begin{array}{r}
{\left[\begin{array}{c}
P(L, \bar{s}) \\
\frac{c}{\mathcal{Q}} Q(0, \bar{s})
\end{array}\right]=\left[\begin{array}{lr}
1 / \operatorname{ch} \bar{s} \varphi(\bar{s}) & -\varphi(\bar{s}) \cdot \operatorname{th} \bar{s} \varphi(\bar{s}) \\
1 / \varphi(\bar{s}) \times \operatorname{th} \bar{s} \varphi(\bar{s}) & 1 / c h \bar{s} \varphi(\bar{s})
\end{array}\right]} \\
{\left[\begin{array}{r}
p(0, \bar{s}) \\
\frac{c}{d} Q(L, \bar{s})
\end{array}\right]}
\end{array}
$$

La variable de Laplace $\bar{s}$ correspond à la variable temps réduit $\bar{t}$

avec

$$
\left.\begin{array}{l}
T=L / c \\
\varphi(\bar{s})=\left[1-\frac{2 J_{1}(j \sqrt{\bar{s} / \tau})}{j \sqrt{\bar{s} / \tau} J_{0}(j \sqrt{\bar{s} / \tau})}\right]^{-1 / 2} \\
\tau=T \times \nu / a^{2}
\end{array}\right\}
$$

$J_{0}$ et $J_{1}$ sont les fonctions de Bessel d'ordre 0 et 1 .

On constate que dans la matrice carrée précédente, ne figurent que $\bar{s}$ et le paramètre $T$.

Il s'en suit que deux canalisations ayant des valeurs de $\tau$ égales et soumises aux mêmes excitations auront des réponses identiques. Moyennant une inversion des termes de la matrice carrée $(1 / \operatorname{ch} \bar{s} \varphi(\bar{s})$ etc.).

L'égalité peut s'écrire alors sous la forme suivante :

$$
\begin{aligned}
& p(L, \bar{t})=\int_{0}^{\bar{t}} A(\bar{t}-u) \frac{\partial p(0, u)}{\partial u} d u- \frac{c}{\bar{\alpha}} \int_{0}^{\bar{t}} B(\bar{t}-u) \frac{\partial q(L, u)}{\partial u} d u \\
& \frac{c}{\alpha} q(0, \bar{t})=\int_{0}^{\bar{t}} C(\bar{t}-u) \frac{\partial p(0, u)}{\partial u} d u+ \\
&+\frac{c}{\bar{\alpha}} \int_{0}^{\bar{t}} A(\bar{t}-u) \frac{\partial q(L, u)}{\partial u} d u
\end{aligned}
$$

Cette formulation montre que si on connait la pressions d'entrée $p(\sigma, u)$ et le débit de sortie $q(l, u)$, on peut connaître la valeur à tout instant de la pression de sortie et du débit d'entrée.

De même, on obtient les relations suivantes:

$$
\begin{aligned}
p(0, \bar{t})=\int_{0}^{\bar{t}} A(\bar{t}-u) \frac{\partial p(L, u)}{\partial u} d u+ \\
+\frac{c}{\mathscr{\alpha}} \int_{0}^{\bar{t}} B(\bar{t}-u) \frac{\partial q(0, u)}{\partial u} d u
\end{aligned}
$$

$$
\begin{aligned}
\frac{c}{\mathcal{Q}} q(L, \bar{t})=-\int_{0}^{\bar{t}} C(\bar{t}-u) \frac{\partial p(L, u)}{\partial u} d u+ \\
+\frac{c}{\mathcal{Q}} \int_{0}^{\bar{t}} A(\bar{t}-u) \frac{\partial q(0, u)}{\partial u} d u
\end{aligned}
$$


de même on constate, que si on connait la pression de sortie et le débit d'entrée, on peut calculer la pression d'entrée et le débit de sortie. On montre que les expressions $A(\bar{t}), B(\bar{t}), C(\bar{t})$ s'expriment analytiquement en fonction du temps et des seules caractéristiques physiques de la ligne.

Les graphes des fonctions $A(\bar{t}), B(\bar{t}), C(\bar{t})$ sont données pour trois valeurs de $\tau$ dans les figures $1 \mathrm{a}, 1 \mathrm{~b}$, 1c.
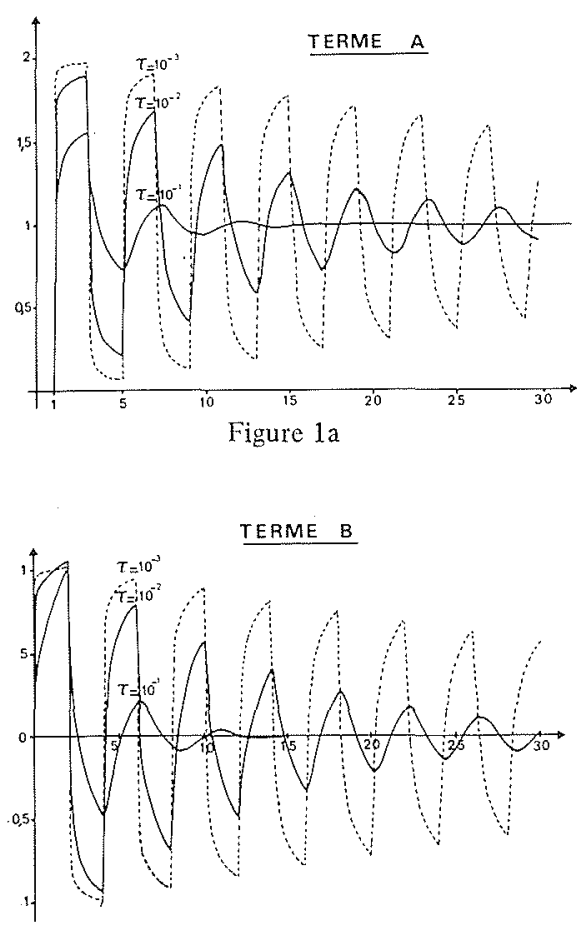

Figure $1 \mathrm{~b}$

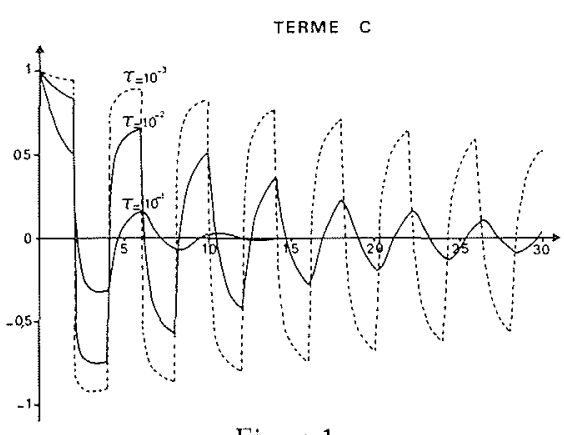

Figure 1c

On constate que ces fonctions ne dépendent que d'un paramètre $\tau$; il caractérise l'amortissement des signaux transitoires dû au frottement visqueux.

Soit deux lignes (1) et (2) différentes mais possédant la même valeur de $\tau$, c'est-à-dire :

$$
\tau_{1}=\frac{L_{1}}{c_{1}} \times \frac{\nu_{1}}{a_{1}^{2}}=\tau_{2}=\frac{L_{2}}{c_{2}} \times \frac{\nu_{2}}{a_{2}^{2}}
$$

La théorie montre que les termes $A(\bar{t}), B(\bar{t}), C(\bar{t})$ sont les mêmes.

Ainsi les valeurs de $p$ et $\frac{c}{\mathscr{Q}} q$ sont identiques à condition que les excitations soient identiques.

\section{Méthode de calcul}

\section{Cas général}

a) Le paramètre de similitue $\tau$ renseigne sur l'importance des phénomènes d'amortissement. Il peut se calculer à l'aide d'abaques.

On peut considérer que $\tau=10^{-2}$ est une valeur moyenne couramment rencontrée ; $\tau=10^{-1}$ représente un amortissement très important et $\tau=10^{-3}$ un amortissement très faible.

Ce paramètre de similitude $\tau$ dépend de 9 paramètres physiques; ces paramètres sont des caractéristiques de l'huile et de la ligne (viscosité, élasticité)

$$
\tau=T \times \nu / a^{2} \quad T=\frac{L}{c} \quad c=\sqrt{K_{e} / \rho}
$$

b) Les deux couples d'équations 10-11 et 12-13 montrent qu'il n'y a que deux combinaisons possibles des conditions aux extrémités d'une ligne : il y a une grandeur physique imposée à chaque extrémité, de ce fait l'autre grandeur se déduit par le calcul.

Soit l'exemple suivant :

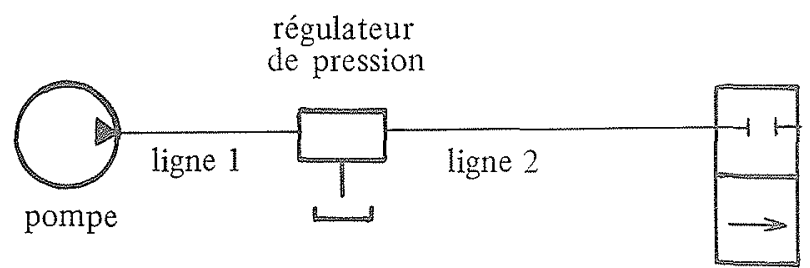

- la pompe impose des pulsations de débit : elles constituent l'entrée imposée de la ligne 1

- le régulateur de pression impose une relation entre le débit et la pression en sortie de la ligne 1 et en entrée de la ligne 2

$P_{1}=Z_{q}(q=$ débit dans le régulateur $)$

- le distributeur fermé en sortie de ligne 2 impose une loi de débit $q_{d}=0$.

Cet exemple montre qu'avant tout calcul, il faut faire un examen détaillé du système pour déterminer :

- les signaux d'entrée

- les impédances de sortie $Z$.

Il faut remarquer que dans le cas de branchement on applique la "Ioi des nœuds", c'est-à-dire que dans le cas de l'exemple cité :

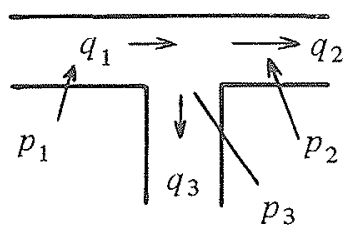

$$
\begin{aligned}
& q_{1}-q_{3}=q_{2} \\
& p_{1}=p_{2}=p_{3}
\end{aligned}
$$

La pression est la même à la sortie de la ligne 1 et à l'entrée de la ligne 2 et du régulateur de pression ; le débit qui sort de la ligne 1 est égal à la somme du débit sortant du régulateur au débit entrant dans la ligne 2.

En fin, on peut appliquer les équations générales des 
lignes (*) ; ces équations se simplifient grâce aux relations établies précédemment ; alors, il reste à effectuer les produits de convolution.

* (Suivant les entrées, on choisit ou le couple (10-11) ou le couple (12-13)).

\section{Cas particulier}

Les figures $2,3,4,5$ illustrent différents cas réalisables expérimentalement.

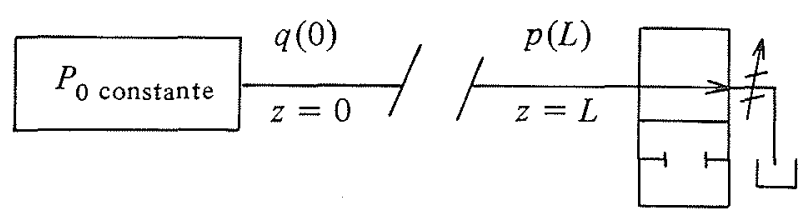

Figure 2

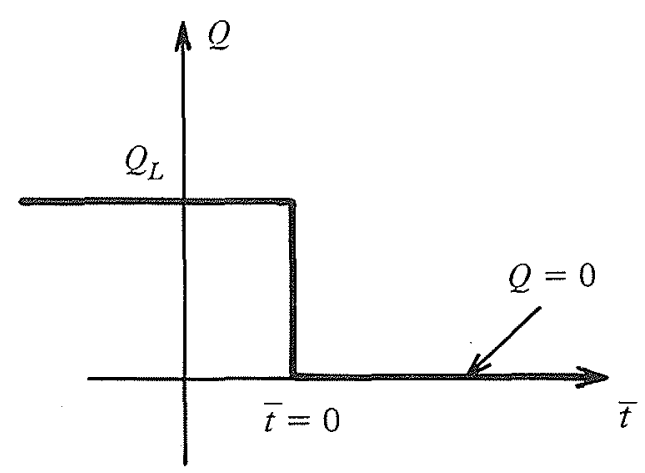

Figure 3
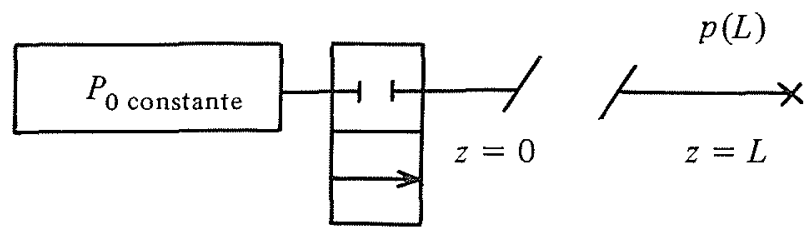

Figure 4

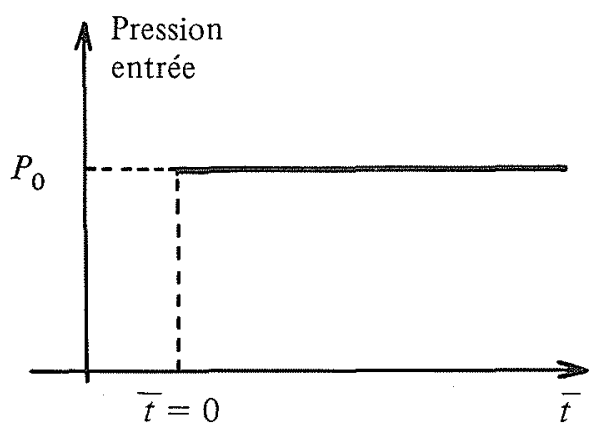

Figure 5

\section{Etude expérimentale}

\section{Banc d'essais}

La source à pression constante est constituée par une pompe alimentant un accumulateur de grande capacité avec régulateurs de pression, les pulsations de pression sont créées par une servovalve.

Ces ouvertures et fermetures brutales sont réalisées par des distributeurs tout ou rien.

Les mesures de pression et de vitesse sont effectuées par des capteurs piezo-capacitifs et des fils chauds pour les mesures instationnaires; les mesures stationnaires sont effectuées par des capteurs piezo-résistifs et des débitmètres à turbine.

\section{Vérification de la similitude}

Il s'agit de vérifier que pour deux lignes différentes, par leur longueur et leur diamètre, mais ayant la même valeur de $\tau$ correspond une réponse identique lorsque le signal d'entrée est le même. La figure 2 montre la bonne corrélation qui existe entre les deux réponses.

\section{Essai en instationnaire}

Par la mesure de certaines pressions ou débit, on obtient l'image des termes $A(\bar{t}), B(\bar{t}), C(\bar{t})$. Les figures 2,3 et 4 montrent la bonne corrélation entre l'expérience et la théorie.

\section{Essai en régime périodique}

Une servovalve impose des pulsations de pression dans une ligne bouchée ou fermée par un robinet.

Pour la ligne fermée : $Z=\infty \quad 1 / Z=0$

$$
P(L, \bar{s}) / P(0, \bar{s})=A(\bar{s})
$$

Pour la ligne ouverte : $Z=\xi$

$$
P(L, \bar{s}) / P(0, \bar{s})=A(\bar{s}) /[1+\xi B(\bar{s})]
$$

Dans le cas de la ligne fermée, le rapport des amplitudes $P(L) / P(0)$ est l'image du terme $A$; dans le cas de la ligne ouverte, c'est l'image d'une combinaison de $A$ et de $B$.

\section{Applications pratiques \\ Phénomènes instationnaires}

Les coups de bélier, tels que ceux vus dans la partie théorique, se rencontrent très rarement dans la pratique, car ils nécessitent le respect des conditions énoncées.

Cependant, il faut remarquer :

- Lors d'un coup de bélier par ouverture, la surpression est au maximum égale au double de la pression imposée à l'entrée.

- Plus $\tau$ est grand, moins les fronts sont raides et plus l'amortissement est rapide. 
Ces termes $B(\bar{t})$ et $A(\bar{t})$ illustrent ces remarques (cf. Fig. 1).

\section{Phénomènes stationnaires}

La figure 6 illustre le cas de la ligne fermée et montre que suivant le type de fluctuations imposées à l'entrée de la ligne (pression ou débit), le maximum de gain en pression est décalé selon le cas.

En effet, on utilise les équations de départ

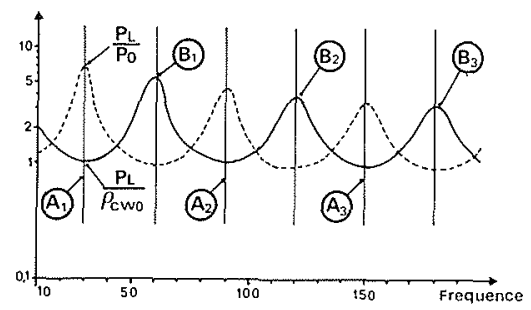

Figure 6. - Fluctuations de pression ou débit dans une ligne fermée

$$
\begin{aligned}
& P(L) / P(0)=A(\bar{s}) /[1+B(\bar{s}) / Z(\bar{s})] \\
& P(L) /\left[\frac{c}{\mathcal{Q}} Q(0)\right]=A(\bar{s}) /[C(\bar{s})+1 / Z(\bar{s})]
\end{aligned}
$$

dans lesquelles on fait $Z(s)=\infty$ car le tuyau est bouché.

$$
P(L) / P(0)=A(\bar{s}) \text { et } P(L) /\left[\frac{c}{\mathscr{Q}} Q(0)\right]=A(\bar{s}) / C(\bar{s})
$$

On montre que $A(s)$ et $A(s) / C(s)$ sont équivalentes en termes réels aux fonctions cosinus et sinus pour les- quelles le maximum de l'une est bien le minimum de l'autre (en valeur absolue).

A propos de ces deux cas particuliers simples, on peut tirer quelques règles générales sur le dimensionnement des lignes.

Une pompe génère des pulsations de débit qui se trouvent amplifiées ou réduites par la ligne située en aval : il faut choisir une longueur telle que le produit de la fréquence de rotation par le nombre de pistons, donne une fréquence qui corresponde à un creux de la courbe 6 , c'est-à-dire les fréquences $A_{1}, A_{2}, A_{3}$. Si l'appareil fournit des pulsations de pression, il faudra par contre choisir les fréquences $B_{1}, B_{2}, B_{3}$.

En effet les fluctuations de pression (qu'on mesure facilement) à la sortie d'une pompe ne sont que le fait de la ligne qui transforme les fluctuations de débit en fluctuations de pression.

\section{Utilisation de l'accumulateur}

Une étude approfondie du fonctionnement de l'accumulateur en antipulsation montre que les variations de la pression $(\Delta P)$ et du débit $(\Delta Q)$ à l'entrée de l'accumulateur sont liées par une relation simple

$$
\Delta P / \Delta Q=\frac{G}{\rho}\left(\frac{\omega}{\omega_{c}}-\frac{\omega_{c}}{\omega}\right)
$$

$$
G=\frac{\gamma P_{1}^{2} T_{0}}{P_{0} V_{0} T_{1} \omega_{c}} \quad \omega_{c}=\sqrt{\frac{\gamma P_{1}^{2} T_{0}}{P_{0} V_{0} T_{1}} \times \frac{\mathscr{Q}^{2}}{m e}}
$$

$P_{1}, T_{1} \quad$ pression température moyenne de fonctionnement

$P_{0}, V_{0}, T_{0}$ pression, volume, température moyenne de gonflage

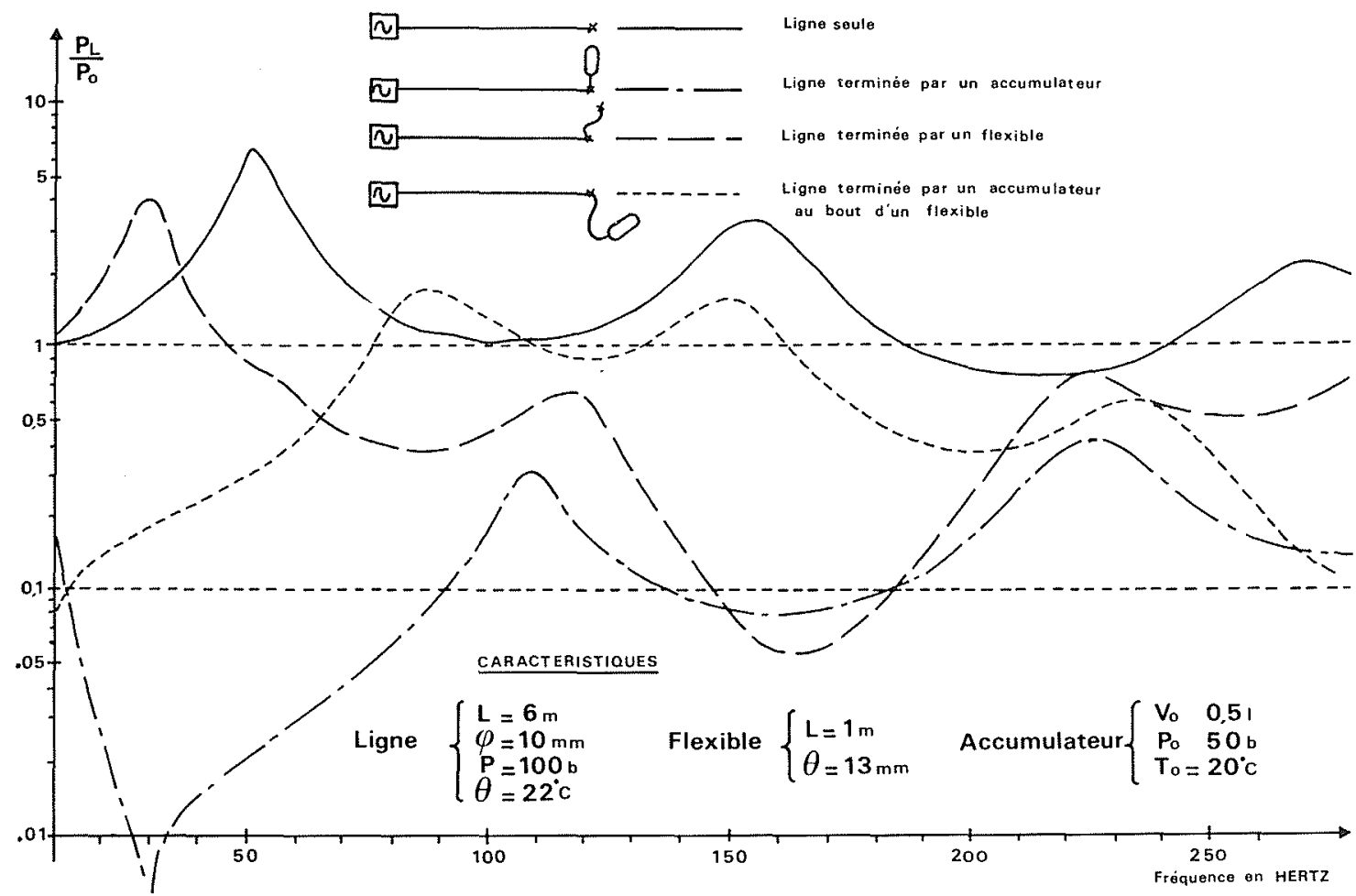

Figure 7. - Courbes expérimentales de la réponse en fréquence d'une ligne terminée de différentes façons. 


$\begin{array}{ll}\gamma & \text { rapport des chaleurs spécifiques } \\ \mathcal{Q} & \text { section de l'organe de séparation } \\ \text { me } & \text { masse équivalente }\end{array}$

On constate que pour $\omega=\omega c, \Delta p / \Delta q$ est nul, c'est-àdire que quelle que soit l'amplitude des variations du débit, les variations de pression sont nulles.

On peut donc tirer des considérations précédentes une règle simple pour le choix de l'accumulateur: sa fréquence d'accord $\omega c$ est égale à la fréquence des pulsations que l'on veut atténuer au maximum.

Cette valeur $\omega c$, calculée à partir des caractéristiques géométriques de l'accumulateur et des conditions de fonctionnement, permet de choisir l'accumulateur adapté : en effet, il n'est pas possible de changer $T_{1}$ et $P_{1}$, il faut donc jouer sur les conditions de précharges $\left(P_{0}, T_{0}\right)$ ou sur les dimensions de l'accumulateur $V_{0}, \mathcal{Q}^{2} / m e$.

Sur la figure 7, on constate l'importance de l'amortis. sement autour de la fréquence d'accord.

Il faut remarquer, que dans ce cas, jusqu'à $200 \mathrm{~Hz}$, l'accumulateur joue un rôle d'amortissement important.

Le cas présenté à la figure 7 est le cas très simple, courant, du tuyau bouché terminé par un accumulateur dans lequel on impose des pulsations de pression; ce cas a l'avantage d'être mathématiquement et expérimen. telement simple et permet donc de vérifier la théorie.

Sur la figure 7, on compare les effets d'un accumulateur au bout du flexible sur une ligne bouchée dans laquelle on génère des pulsations de pression ; on constate :

- seul l'accumulateur fournit une très bonne atténuation jusqu'à $100 \mathrm{~Hz}$

- entre 100 et $250 \mathrm{~Hz}$, l'accumulateur et le flexible ont des effets semblables
- l'association accumulateur et flexible en série est très mauvaise

- en effet, on augmente la masse de l'huile de l'accumulateur de la masse d'huile contenue dans le tuyau de sorte que l'ensemble a une forte inertie, donc une fréquence d'accord basse.

L'accumulateur peut être caractérisé par deux constantes pour chaque point de fonctionnement : le gain et la fréquence d'accord.

On constate avec quelle précision la méthode de calcul permet de déterminer la réponse de l'ensemble ligne + accumulateur.

\section{Phénomènes instationnaires complexes}

Le CETIM dispose d'un programme de simulation qui permet de résoudre les systèmes d'équations différencielles et d'équations implicites : la représentation matricielle de la ligne étant intégrée dans ce programme, il est alors possible de résoudre toutes les conditions aux extrémités de la ligne linéaire ou non.

Dans l'exemple de la figure 8 , à l'entrée de la ligne, on dispose :

- d'équations algébriques qui représentent la loi de débit de la pompe fonction du temps

- d'un système d'équations différencielles qui régissent le fonctionnement de l'accumulateur.

A la sortie de la ligne, on dispose :

- d'une relation d'une perte de charge liant la pression et le débit.

Sur la figure 8 , on a représenté un démarrage de pompe volumétrique avec et sans accumulateur. Cette pompe débite dans une ligne terminée par une résistance. On observe l'effet bénéfique de l'accumulateur sur l'amplitude du coup de bélier.

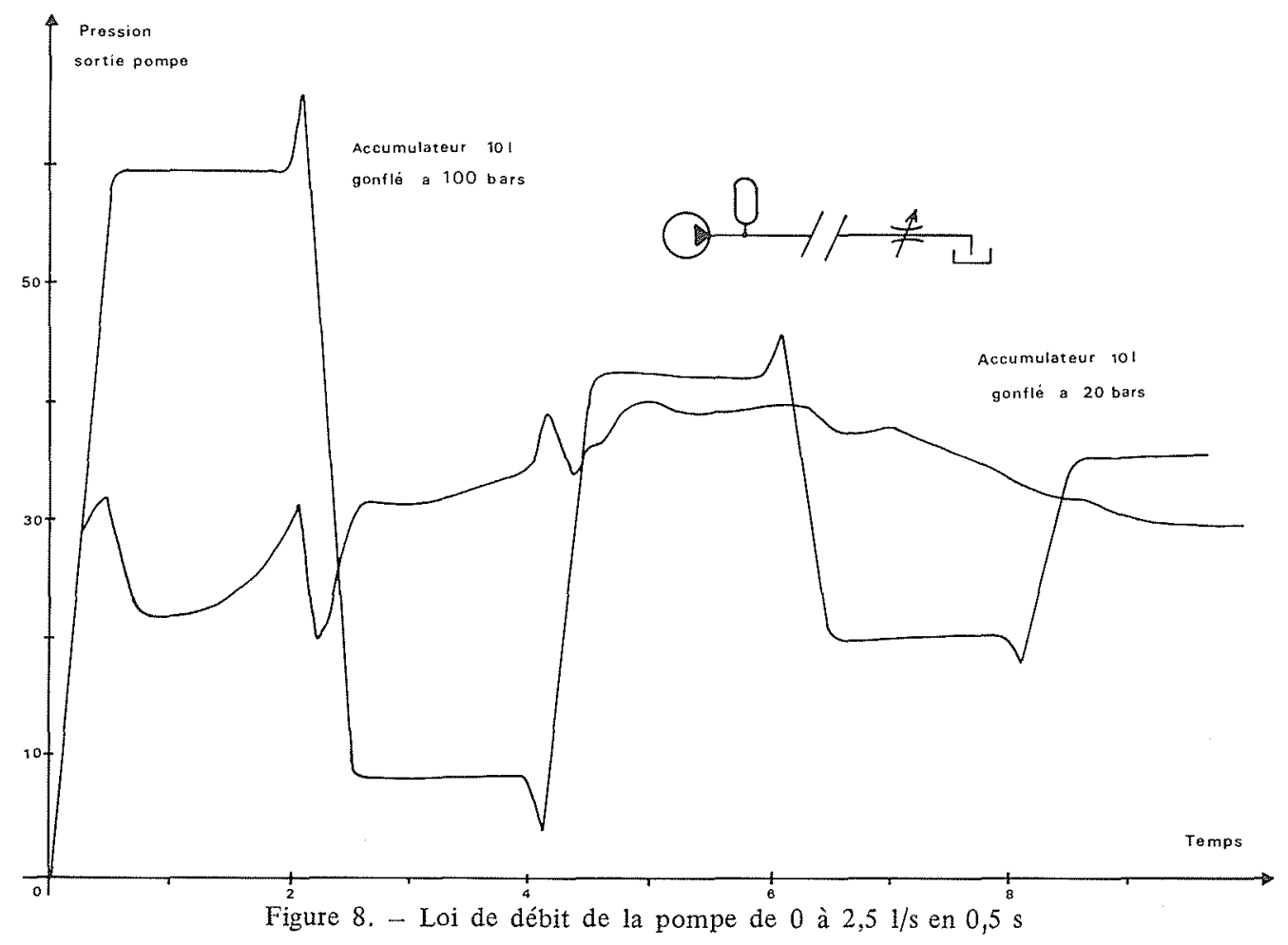




\section{Conclusion}

Les méthodes de calcul présentées permettent de simuler le comportement dynamique des lignes et des canalisations hydrauliques.

Ces méthodes sont simples d'emploi et aisément programmables sur ordinateurs. Elles offrent l'avantage d'être applicables:

- quelles que soient la nature des appareils hydrauliques placés en série, la seule condition étant de connaitre la modélisation mathématique de chaque appareil, - quelle que soit la forme du signal d'excitation, que celui-ci soit un signal de débit ou de pression.

L'amortissement propre dû aux fluides est pris en compte de manière rationnelle, en partant des équations générales de la Mécanique des Fluides, ce qui permet par exemple d'obtenir, avec une très bonne précision, l'amplification d'un signal de pression lors- qu'une ligne entre en résonnance. L'influence de la compressibilité de l'air dissous dans l'huile, du comportement élastique ou visco-élastique, des effets thermiques est aussi pris en compte. Enfin, ces méthodes de calcul peuvent être utilisées pour l'étude de systèmes hydrauliques non linéaires.

Des exemples d'application ont été indiqués pour l'étude des coups de bélier, des pulsations de pression dans les canalisations, le dimensionnement, le positionnement et l'optimisation des accumulateurs.

De nombreux autres cas pourraient être étudiés à l'aide de ces méthodes de calcul.

D'une manière générale, cette méthode est adaptée au calcul rationnel des problèmes de vibrations mécaniques des canalisations, l'excitation étant d'origine hydraulique, à l'étude du bruit des appareils hydrauliques et de sa propagation dans les canalisations et appareils.

\section{Discussion}

M. CHEVALIER, - Nous allons ouvrir la discussion. Vous nous avez développé une méthode mais ceci étant, sous quelle forme cette méthode se présente-t-elle ? Comment est-elle utilisable ? Quel temps de calcul nécessite-t-elle ? Quels problèmes perme t-elle de traiter?

Vous ave $z$ présenté des exemples intéressants mais les circuits de transmission sont quelquefois beaucoup plus complexes. Peuton traiter des schémas plus complexes que ceux que vous avez présentés?

M. LALLEMENT. - On peut simuler les fonctionnements de toutes installations lorsqu'on connait les modèles et les coefficients caractéristiques de chacun des appareils de l'installation. Dans le domaine des transmissions hydrostatiques les modèles sont connus mais on doit déterminer expérimentalement la valeur des coefficients. Ceci est fait en utilisant un programme d'identification dont le principe est le suivant :

- on compare les résultats de mesure et les résultats donnés par

le modèle avec certains coefficients, un programme de mini-

misation de l'écart moyen permet d'atteindre la valeur correcte des coefficients.

Les schémas d'installations complexes peuvent être simulés moyennant des temps de calcul plus longs.

M. CHEVALIER. - La personne qui fait le plan d'installation dispose-t-elle do tous les éléments nécessaires correspondant à chaque élément du circuit ?

M. LALLEMENT. - Le concepteur possède une grande partie des données et connait le cahier des charges qui lui est imposé. Avec ces données, le schéma de l'installation et le programme de calcul, il peut par simulation vérifier que les performances sont bien celles attendues. S'il constate que les résultats ne sont pas satisfaisants. il doit modifier son schéma ou les données et effectuer une nouvelle simulation. Les paramètres inconnus du concepteur sont à identifier par un essai séparé.

M. CHEVALIER. - Il est évident qu'il y aura à faire une expérimentation par appareil, ce qui risque de coûter plus cher que de faire l'expérimentation sur l'installation totale.
M. PATIN, - J'aurai trois questions à poser, la première au sujet de l'accumulateur :

. Quel est le rôle de laccumulateur pour écrêter le coup de bélier, n'est-il pas négligeable quand on fonctionne à pleine pression?

. Comment peut-on générer des variations de pression sans générer des variations de débit?

. Quelles vitesses sont admissibles dans les tuyauteries de transmissions hydrauliques?

M. LALLEMÉNT. - L'accumulateur peut-être déterminé pour écrêter les coups de bélier de manière optimum pour une pression donnée de fonctionnement. Si celle-ci change l'écretage est moins bon.

M. PATIN. - Je vois très bien comment on peut faire des variations cycliques de débit, mais comment faites-vous des variations cycliques de pression en régime permanent ?

M. LALLEMENT. - Les variations cycliques de pression sont générées par une servo-valve qui est un distributeur qu'on asservit électroniquement à la variation de pression désirée.

M. PATIN. - Par l'intermédiaire d'une variation de volume?

M. LALLEMENT. - Oui bien sûr.

M. BERGERON. - Parler d'une variation de débit c'est parler d'une variation de pression, les deux sont conjuguées.

M. LALLEMENT. - Une pompe volumétrique est un générateur de pulsations de débit, mais on ne mesure que les variations de pression consécutives à cette puisation đe débit.

M. PATIN. - Vous voulez dire que la variation de pression n'est qu'une conséquence?

M. TOURRET. - Une source de pulsation de pression est également une source de pulsation de débit. Sur le plan pratique, c'est la source qui impose les types de pulsation : ainsi une pompe à piston impose essentiellement une variation de débit et c'est l'impédance du circuit qui impose la valeur des pulsations de pression. 
M. BONNIN. - Vous avez formulé au début de votre exposé l'hypothèse d'une masse de paroi (tuyauterie) négligeable, ce qui, à hau te pression, paraît très loin de la réalité.

Je pense que la faible influence de cette masse est due au rapport entre la vitesse de la paroi et la fluctuation de vitesse du fluide, lui-même proportionnel au rapport - élevé - entre le module d'élasticité de la paroi et le module de compressibilité du fluide.

M. LALLEMENT. - La vitesse du son dans l'acier est quatre fois supérieure à celle du son dans l'huile. Nous avons des tuyauteries très épaisses de sorte que les déplacements sont faibles.

M. BONNIN. - Quelle loi de perte de charge avez-vous adoptée pour l'écoulement visqueux non permanent (fréquences jusqu'à $200 \mathrm{~Hz}$ ) ?

M. LALLEMENT. - J'ai gardé le terme de "viscosité", multiplié par la dérivée seconde.

M. BONNIN. - Vous avez pris les mêmes pertes de charge?

M. LALLEMENT. - Je n'ai pas fait d'hypothèse. J'ai gardé les termes des équations de Navier-Stockes.

M. BERHAULT. - Le modèle mathématique présenté ici a-t-il été également utilisé pour la prédiction du comportement d'une transmission hydrostatique (pompe, ligne, moteur) ?

M. LALLEMENT. - Nous ne l'avons pas testé sur une transmission, nous l'avons testé sur un circuit de pompage où on avait plusieurs pompes qui débitaient dans l'installation à des endroits différents.

En faisant la superposition des modes vibratoires, les résultats obtenus ont été excellents.

Ceci a été fait dans le cadre de l'assistance technique au CETIM, je ne peux pas vous en parler davantage.

M. CHEVALIER, - Dans ce cas vous n'êtes plus dans les hypothèses d'écoulement laminaire.

Si vous avez pris une installation industrielle avec différentes pompes qui alimentent un circuit, vous n'étiez plus dans vos hy pothèses de départ?

M. LALLEMENT. - Même dans cet exemple de type industriel, les résultats de calcul ont été confirmés par les mesures.

$M$. CORBELLINI. - Je voudrais que vous nous indiquiez les conditions d'accès aux modèles mathématiques.

M. LALLEMENT. - Vous pouvez soumettre votre problème complet au CETIM ou utiliser vous-même le programme qui ne demande pas de connaissances informatiques.

M. CORBELLINI. - Dans le cas d'un modèle écrit quelle est la participation financière?

M. LALLEMENT. - Tout dépend du problème posé et du temps de calcul.

M. - PATIN, - Etes-vous arrivé à une conclusion en ce qui concerne les vitesses de circulation du fluide dans les conduites des transmissions classiques, c'est-à-dire avec des huiles de viscosité également classique?

Pouvez-vous citer un chiffre raisonnable?

Le problème du dimensionnement des conduites est très important dans l'établissement d'une transmission.

M. LALLEMENT. - S'il n'y a pas de problème de coup de bélier, les canalisations sont généralement imposées par les raccordements des pompes.

Pour l'instant, les clients du CETIM sont des sociétés qui ont des problèmes de mise au point. Ils ont une installation qui fonctionne mal ; notre rôle est d'assurer un fonctionnement normal au moindre coût possible.

M. SCHMITT, - Quelle est la méthode utilisée pour mesurer la vitesse?

M. LALLEMENT. - Les mesures ont été faites dans l'huile avec des "fils chauds".

M. SCHMITT. - Quelle est la méthode expérimentale utilisée pour caractériser un élément hydraulique a priori inconnu, et quelle est l'importance de l'expérimentation?

M. LALLEMENT. - Tout dépend du modèle qu'on a et du nombre de paramètres qu'on peut identifier.

M. SCHMITT. - La caractérisation est obtenue en plaçan l'élément sur un circuit hydraulique et en mesurant sa réponse à des ouvertures ou fermetures de vannes.
Pour un accumulateur, ces essais nécessitent quelques heures, et au moins quinze jours pour une pompe.

M. $L A L L E M E N T$, - Tout dépend du nombre de coefficients à identifier pour cette pompe.

M. WARTELLE. - Tout dépend de la précision qu'on attend du modèle. Il existe des modèles très simplifiés, avec peu de paramètres, et des modèles beaucoup plus compliqués prenant en compte par exemple la compressibilité, les différents frottements secs et lubrifiés.

M. CHEVALIER - Vous parlez de pompes volumétriques?

M. WARTELLE, - Oui.

M. LALLEMENT. - Pour un accumulateur deux coefficients sont à identifier. Un seul essai est nécessaire.

M. CHEVALIER, - Quels types d'expériences ?

M. LALLEMENT. - Des ouvertures et fermetures de vannes et des mesures de pression par exemple.

M. VALIBOUSE. - Est-ce que vous intégrez dans votre programme les réactions d'un appareil monté sur la boucle et, s'il y a aussi interaction; faites-vous les calculs itératifs correspondants?

D'autre part, tenez-vous compte : de l'influence de l'air sur la compressibilité ; des échanges gaz libres-gaz dissous pendant les phénomènes instationnaires?

M. LALLEMENT. - Pour répondre à la première question, il est bien évident que, si,mathématiquement, vous ne tenez pas compte d'un phénomène et que, physiquement, c'est ce phénomène qui intervient, le modèle ne marchera pas.

$M$. WARTELLE. - Pour préciser la réponse de $\mathrm{M}$. LALLEMENT, disons que pour un appareil du genre clapet, on peut avoir deux types de modèle : un modèle stationnaire quand on veut étudier des problèmes très lents; un modèle beaucoup plus compliqué, valable en instationnaire, tenant compte de l'inertie du clapet, des réactions du fluide sur le clapet : le calcul est effectivement beaucoup plus long.

M. LALLEMENT. - Lors des vérifications du coup de bélier, on a constaté que la compressibilité de lhuile variait au cours de la journée.

M. DIVENOT, - Votre méthode - je parle de l'équation de base - est-elle supérieure à des méthodes déjà connues?

M. LALLEMENT, - La méthode proposée permet le calcul des circuits indépendamment de la nature des organes d'entrée et de sortie pourvu que leur impédance ou leur modèle mathématique soit connu. Elle tient compte de l'influence de la viscosité.

M. KHALETZKY. - Vous supposez bien que la perte de charge est proportionnelle à la vitesse ?

M. WARTELLE. - En régime instationnaire, il y a modification du profil des vitesses qui fait que, même en supposant le régime laminaire, on n'obtient pas à hau te fréquence une perte de charge proportionnelle au débit. Les frottements fluides sont plus importants à haute fréquence.

M. LALLEMENT. - Il est sûr que ces phénomènes sont plus importants dans l'huile que dans l'eau, puisque la viscosité est plus importante.

M. CORBELLINI, - Je voudrais savoir si vous travaillez sur une fonction de transfert permettant d'associer la pulsation hydraulique au bruit aérien; c'est un problème qu'on retrouve de plus en plus, en particulier lorsqu'un tube traverse une cloison métallique.

M. LALLEMENT. - Vous évoquez le problème du transfert entre le bruit que l'on entend à l'oreille et les pulsations de débit ou de.pression que l'on peut mesurer dans les tuyauteries. C'est un problème d'acousticien.

M. CHEVALIER. - Je remercie M. LALLEMENT. Sa communication a attiré des questions nombreuses, elle a montré que toute une gamme de professionnels travaillant dans le domaine des transmissions hydrauliques avait besoin d'un certain apport qu'apparemment ils n'ont pas trouvé dans ce qui existait. Ils ont été amenés à bâtir quelque chose qui leur est propre.

Les exemples qui nous ont été présentés ont fait apparaître un certain nombre d'avantages que M. LALLEMENT nous a soulignés. 